\title{
Study of the Hindrance Effect in Sub-barrier Fusion Reactions
}

\author{
Masahiro Notani ${ }^{\mathrm{a}}$, P. Davies ${ }^{\mathrm{b}}$, B. Bucher ${ }^{\mathrm{a}}$, X. Fang ${ }^{\mathrm{a}}$, L. Lamm , C. Ma $^{\mathrm{a}}$, E. Martin ${ }^{\mathrm{b}}$, \\ W. Tan ${ }^{\mathrm{a}}$, X.D. Tang ${ }^{\mathrm{a}}$, S. Thomas ${ }^{\mathrm{ab}}$ and C.L. Jiang ${ }^{\mathrm{c}}$ \\ ${ }^{a}$ Department of Physics, University of Notre Dame, Notre Dame, IN 46556, U.S.A. \\ bUniversity of Surrey, Guildford, Surrey, GU2 7XH, U.K. \\ cPhysics Division, Argonne National Laboratory, Argonne, IL 60439, U.S.A.
}

We have measured the fusion cross sections of the ${ }^{12} \mathrm{C}\left({ }^{13} \mathrm{C}, p\right){ }^{24} \mathrm{Na}$ reaction through off-line measurement of the $\beta$ decay of ${ }^{24} \mathrm{Na}$ using the $\beta-\gamma$ coincidence method. Our new measurements in the energy range of $E_{c . m} .=2.6-3.0 \mathrm{MeV}$ do not show an obvious $S$ factor maximum but a plateau. Comparison between this work and various models is presented.

Heavy-ion fusion reactions between light nuclei such as carbon and oxygen isotopes have been studied because of their significance for a wide variety of stellar burning processes, such as supernovas and superbursters [1]. It has been suggested that stars with intermediate mass or 8-10 of solar mass may cause a shock wave by carbon ignition under electron degeneracy conditions and is observed as a supernova explosion [2]. Superbursts on accreting neutron stars have been related to carbon burning, for which accurate reaction rates are required to understand the theoretical models [3]. The temperatures for these carbon burning processes range from 0.8 to $1.2 \times 10^{9} \mathrm{~K}$, corresponding to $E_{c . m}$. $=$ $1-3 \mathrm{MeV}$.

The fusion reactions have only been measured in the laboratory down to energies that are much higher than those of astrophysical interest so far. Optical models have been widely used to fit the experimental data, and to estimate the reaction cross sections or the astrophysical $S$-factor: $S(E)=E \sigma(E) \exp (2 \pi \eta)$ at the lower energies by extrapolation. Much effort has been devoted to measure the fusion cross section such as ${ }^{12} \mathrm{C}+{ }^{12} \mathrm{C}$ reaction at astrophysical energies by charged particle and $\gamma$-ray spectroscopy [4]. However, the extrapolation is still needed to estimate the reaction rate for nucleosynthesis simulation, and the extrapolation is difficult due to the large resonant structure.

Recently, a hindrance effect in low-energy heavy-ion fusion reactions was discovered in medium-heavy nuclei system, such as ${ }^{64} \mathrm{Ni}+{ }^{64} \mathrm{Ni}$ [5], and the systematics show that the hindrance could affect the astrophysically important fusion reactions [6]. Therefore the measurement of fusion cross sections in low-energy light-ion fusion reactions is required to verify the hindrance effect.

We report an experiment to measure the cross section of ${ }^{13} \mathrm{C}+{ }^{12} \mathrm{C}$ reaction at extreme sub-barrier energies. This reaction was investigated because unlike the ${ }^{12} \mathrm{C}+{ }^{12} \mathrm{C}$ reaction, it does not show the large resonant structure but relatively smooth excitation function. 


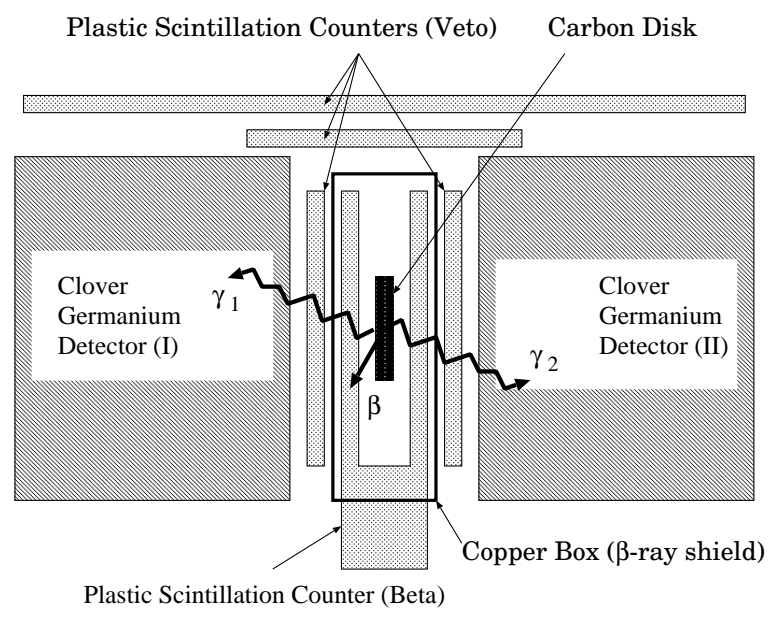

Figure 1. Schematic view of the experimental setup.

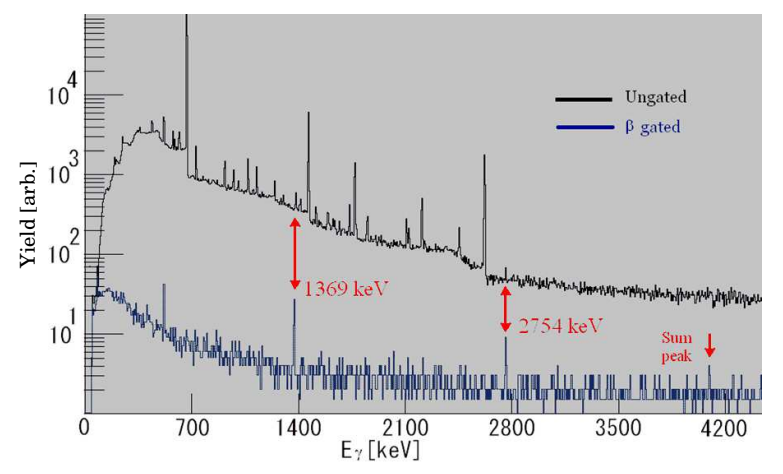

Figure 2. Comparison of two $\gamma$-ray spectra obtained with and without the $\beta$-ray gate.

The ${ }^{13} \mathrm{C}$ beam was provided by the 10-MV FN Tandem Van de Graaff Accelerator at the University of Notre Dame. To obtain an intense beam, we used a gas stripper system in the accelerator. The charge-state distribution of ${ }^{13} \mathrm{C}$ ions after the gas stripper was studied and $2^{+}$state ions were selected. The beam energies were determined by measuring the magnetic field in the analyzing magnet after the accelerator. The ${ }^{13} \mathrm{C}$ beam was impinged on a 1-mm thick carbon target.

The cross section for the ${ }^{12} \mathrm{C}\left({ }^{13} \mathrm{C}, p\right){ }^{24} \mathrm{Na}$ reaction has been determined through the measurement of $\beta$-decay rate of ${ }^{24} \mathrm{Na}\left(T_{1 / 2}=14.9 \mathrm{hrs}\right)$ using the $\beta$ - $\gamma$ coincidence method. The irradiated carbon target was transported to a detection system for the $\beta$-decay measurement. Figure 1 shows the experimental setup of the detector array for the $\beta$ - $\gamma$ coincidence measurement. The carbon target was surrounded by plastic scintillation counters to detect the $\beta$ rays from ${ }^{24} \mathrm{Na}$. Following the $\beta$ decay of ${ }^{24} \mathrm{Na}$, two $\gamma$ rays $(1369 \mathrm{keV}$ and $2754 \mathrm{keV}$ ) are emitted from the excited ${ }^{24} \mathrm{Mg}$. The $\beta$-delayed $\gamma$ rays were detected by two Ge clover detectors. The typical spectra are shown in Fig. 2, In addition, cosmic rays were vetoed by an array of four plastic scintillation counters. The detection system was shielded with a 7 -cm thick lead.

The thick target yield was obtained by normalizing the efficiency corrected $\beta$-gated $\gamma$-ray yield to the total incident ${ }^{13} \mathrm{C}$ charge. From the thick-target excitation function, the differential yield $d Y / d E$ was determined and the cross section was calculated using the following equation,

$\sigma(E)=\frac{M_{T}}{f N_{A}} \frac{d Y}{d E} \frac{d E}{d(\rho X)}$,

where $M_{T}$ is the molecular weight of the target, $f$ is the molecular fraction of the target nucleus of interest, $N_{A}$ is Avogadro's number and $d E / d(\rho X)$ is the stopping power given by the $S R I M$ code [4].

In order to deduce the total cross section for compound nucleus formation or the fusion reaction cross section of ${ }^{12} \mathrm{C}+{ }^{13} \mathrm{C}$, the theoretical branching ratio for the proton emission 


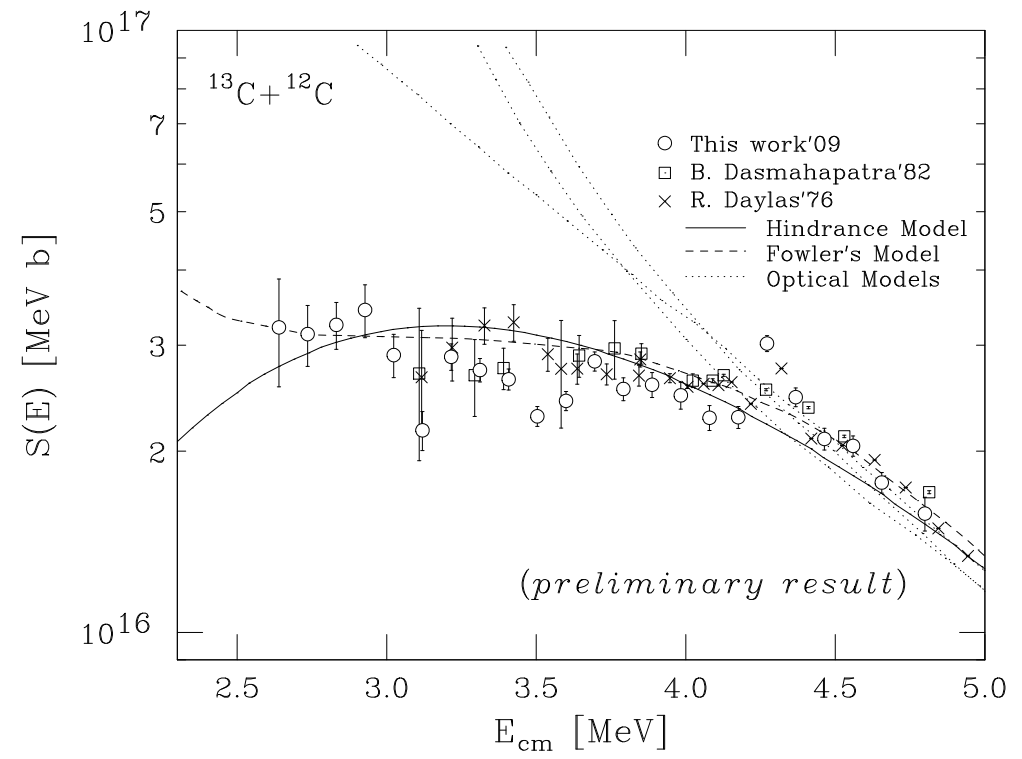

Figure 3. Astrophysical $S$ factor $S(E)$ as a function of the center-of-mass energy $E$, derived from the fusion cross sections, obtained from the previous experiments [7,8] and this work.

channel was used as correction factor, as discussed in Ref. [7].

Figure 3 shows the astrophysical $S$-factor obtained from this experiment. The result agrees with that of the two previous measurements in the energy region from 3.2-5.0 MeV. We have obtained 5 new data points in the range of $E_{c . m} .=2.6-3.0 \mathrm{MeV}$, which overlaps the important energy range for the carbon burning. Within this energy range, our data do not show an obvious $S$-factor maximum but a plateau. The optical models (dotted curves) with Woods-Saxon type potential [9] reproduces the experimental data only at energies above $4 \mathrm{MeV}$. The Fowler's model (dashed curve) which employs a repulsive potential [7,10] explains the plateau $\left(E_{c . m} .<4 \mathrm{MeV}\right)$ and predicts an increase at very low-energy region $\left(E_{c . m}\right.$. $\left.<2.5 \mathrm{MeV}\right)$. The hindrance model (solid curve) [6] also shows a good agreement to the experimental data and predicts a decrease at lower energies. It should be noted that the parameters of hindrance model were slightly modified by fitting with the present data. In order to check the difference between the extrapolations from Fowler's model and hindrance model, measurements at even lower energies are urgently needed.

\section{REFERENCES}

1. L.R. Gasques et al., Phys. Rev. C 72 (2005) 025806.

2. E. García-Berro et al., Astrophys. J. 485 (1997) 765.

3. A. Cumming et al., Astrophys. J. 646 (2006) 429.

4. L. Barron-Palos et al., Nucl. Phys. A779 (2006) 318.

5. C.L. Jiang et al., Phys. Rev. Lett. 89 (2002) 052701; 93 (2004) 012701.

6. C.L. Jiang et al., Phys. Rev. C 75 (2007) 015803; 79 (2009) 044601.

7. R.A. Dayras et al., Nucl. Phys. A265, 153 (1976).

8. B. Dasmahapatra, B. Cujec and F. Lahlou, Nucl. Phys. A384 (1982) 257.

9. D.J. Crozier and J.C. Legg, Phys. Rev. Lett. 33 (1974) 782.

10. W.A. Fowler et al., Ann. Rev. Astronomy and Astrophys. 13 (1975) 69. 